OPEN ACCESS

Edited by:

Jinhui Wu,

Sichuan University, China

Reviewed by:

Renato Gorga Bandeira De Mello,

Federal University of Rio Grande do

Sul, Brazil

Haixiong Lin,

Guangzhou University of Chinese

Medicine, China

Qinxiu Zhang,

Chengdu University of Traditional

Chinese Medicine, China

*Correspondence:

Guoheng $\mathrm{Hu}$

huguoheng@hnucm.edu.cn

Specialty section:

This article was submitted to

Geriatric Medicine,

a section of the journal

Frontiers in Medicine

Received: 10 August 2021 Accepted: 04 November 2021 Published: 06 December 2021

Citation:

Zhang T, Ou L, Chen Z, Li J, Shang Y and Hu G (2021) Transcutaneous

Electrical Acupoint Stimulation for the Prevention of Postoperative Cognitive Dysfunction: A Systematic Review and Meta-Analysis. Front. Med. 8:756366. doi: 10.3389/fmed.2021.756366

\section{Transcutaneous Electrical Acupoint Stimulation for the Prevention of Postoperative Cognitive Dysfunction: A Systematic Review and Meta-Analysis}

\author{
Tiantian Zhang ${ }^{1,2}$, Liang $\mathrm{Ou}^{3}$, Zehua Chen ${ }^{4}$, Jiamin $\mathrm{Li}^{2}$, Yan Shang ${ }^{2}$ and Guoheng $\mathrm{Hu}^{1 *}$ \\ ${ }^{1}$ Department of Neurology, The First Hospital of Hunan University of Chinese Medicine, Changsha, China, ${ }^{2}$ The Graduate \\ School, Hunan University of Chinese Medicine, Changsha, China, ${ }^{3}$ Department of Orthopedics, The Second Affiliated \\ Hospital of Guizhou University of Traditional Chinese Medicine, Guiyang, China, ${ }^{4}$ The Fifth Clinical Medical College, \\ Guangzhou University of Chinese Medicine, Guangzhou, China
}

Background: No specific treatment is available for postoperative cognitive dysfunction (POCD). Recently, interest in the prevention of POCD during the perioperative period has increased. Although some studies suggest that transcutaneous electrical acupoint stimulation (TEAS) may be beneficial, the relevant evidence remains uncertain.

Objective: To evaluate the preventive effects of TEAS on POCD.

Methods: Seven databases including PubMed, EMBASE, CENTRAL, China National Knowledge Infrastructure (CNKI), Chinese Scientific Journal Database (VIP), Wanfang Database, and Chinese Biomedical Literature Database (CBM) were electronically searched up to April 2021. Two reviewers independently selected the studies, collected data, and assessed the risks of bias and grading of recommendations, assessment, development, and evaluations certainty of the evidence. A meta-analysis of the incidence of POCD, cognitive function score, pain, adverse reactions, and length of hospital stay after surgery was also performed.

Results: Twenty-nine randomized controlled trials with 1,994 participants were included. The results of the meta-analysis showed that the TEAS group has a significantly lower incidence of $\mathrm{POCD}$ compared with the control group on postoperative days 1 [OR $=0.33$ (95\%Cl: 0.23, 0.47); $p<0.001, R^{2}=0 \%$, moderate certainty], 3 [OR $=0.38(95 \% \mathrm{Cl}:$ $0.29,0.50) ; p<0.001, l^{2}=0 \%$, low certainty], and 7 [OR $=0.51$ (95\% Cl: $\left.0.32,0.81\right)$; $p=0.005, l^{2}=0 \%$, low certainty] but not on day 5 ( $p>0.05$, low certainty). Moreover, TEAS improved the Mini-Mental State Examination scores on postoperative days 1, 3, and 7 [MD $=2.44(95 \% \mathrm{Cl}: 1.61,3.27) ; p<0.001, l^{2}=93 \%$, low certainty]; [MD $=2.07$ (95\%Cl: 1.53, 2.62); $p<0.001, l^{2}=87 \%$, low certainty]; and [MD = 0.49 (95\%Cl: 0.18 , $0.79) ; p=0.002, I^{2}=21 \%$, low certainty], respectively, but not on day 5 ( $p>0.05$, very low certainty). TEAS promoted a postoperative analgesic effect within $24 \mathrm{~h}$ after surgery. Furthermore, patients receiving TEAS showed a lower incidence of postoperative nausea and vomiting and a shorter hospital stay. 


\begin{abstract}
Conclusions: Limited evidence suggests that the application of TEAS in the perioperative period is associated with a reduced POCD rate and a protected early postoperative cognitive function.
\end{abstract}

Keywords: transcutaneous electrical acupoint stimulation, postoperative cognitive dysfunction, prevention, cognitive function, systematic review, meta-analysis

\section{INTRODUCTION}

Postoperative cognitive dysfunction (POCD) is a syndrome with prolonged cognitive impairment, which is characterized by limitations in memory, intellectual ability, and executive function after surgery. This condition is distinct from delirium and dementia (1). An estimated 312.9 million surgical procedures were performed worldwide in 2012, displaying an increase of $38 \%$ over the previous 8 years (2). Furthermore, with growing populations and increasing lifespan, the number of surgeries performed annually is likely to continue to increase (3). Cognitive dysfunction following a surgical procedure is one of the most common complications in the elderly, with estimated incidence rates of $20-50 \%$ at 3 months post-cardiac surgery and $5-55 \%$ after other major surgeries $(4,5)$. A higher 1-year mortality rate increased length of stay and cost, and premature withdrawal from the workforce have been observed in patients who experienced POCD. POCD is also associated with an increased risk of dementia and may lead to chronic neurodegeneration, particularly in the case of repeated surgery (6-8). Age, education level, infection, and preexisting cognitive disorders have been associated with cognitive decline after surgical procedures $(4,7,9$, 10). To date, there remains no available strategy for the treatment of POCD. Therefore, anesthesiologists and surgeons have sought to develop an approach to reduce the morbidity of POCD. As the occurrence of POCD may be multifactorial, the prevention methods are correspondingly multidisciplinary $(8,11,12)$.

Acupuncture, as traditional alternative medicine, has been used to treat diseases and relieve pain for thousands of years in Asia. Transcutaneous electrical acupoint stimulation (TEAS) is a combination of transcutaneous electrical nerve stimulation (TENS) and traditional Chinese acupuncture that has been widely accepted and applied worldwide (13). Previous studies have shown the beneficial effects of TEAS for various aspects, such as reducing intraoperative opioid use and postoperative nausea and vomiting (PONV), relieving pain, and improving postoperative cognitive function (14-17). To our knowledge, few studies have systematically examined the efficacy and safety of TEAS for the prevention of POCD based on the Preferred Reporting Items for Systematic reviews and Metaanalyses (PRISMA) guidelines. Therefore, we evaluated the preventive effect of TEAS on POCD to provide evidence for clinical practice by reviewing all currently available randomized controlled trials (RCTs).

\section{METHODS}

This systematic review and meta-analysis are reported in accordance with the PRISMA guidelines (18) and registered on the Open Science Framework (https://osf.io/ bq4v2, doi: 10.17605/OSF.IO/BQ4V2).

\section{Databases and Search Strategy}

We searched PubMed, EMBASE, Cochrane Central Register of Controlled Trials (CENTRAL), China National Knowledge Infrastructure (CNKI), Chinese Scientific Journal Database (VIP), Wanfang Database, and Chinese Biomedical Literature Database (CBM) from the dates of inception of the databases until April 2021 without any language restrictions. The search strategy used medical subject's headings (MeSH) terms in combination with free-text, such as "Cognitive Function," "Cognitive Dysfunction," "Cognition," "Cognitive Impairments," "Postoperative Cognitive Complications," "Transcutaneous Electrical Acupoint Stimulation," "Acupuncture Points," and "Electric Stimulation," etc. The detailed search strategy is described in Supplementary File 1.

\section{Selection Criteria}

This review focused on RCTs that were less prone to confounding bias by indication $(19,20)$. The eligibility criteria for study selection were as follows: (1) Participants aged 18 years and above who underwent surgery and anesthesia and showed no abnormal cognitive function in preoperative assessment; (2) the intervention group was treated with TEAS, alone or combined with other therapies; (3) the control group (CG) was treated with a sham intervention, with no treatment, or other therapies; and (4) no limit to the type of operation. We excluded studies with (1) participants diagnosed with POCD, (2) trials that used needles, such as electroacupuncture, body needle, auricular needle, etc., and (3) articles published using the same data sets.

\section{Outcome Measures}

The primary outcomes were the incidence of POCD and cognitive function scores assessed by any definition given in the original study. The secondary outcomes included postoperative pain, adverse reactions, and length of hospital stay.

\section{Data Extraction and Quality Assessment}

Two investigators (JML and YS) independently extracted data from eligible studies and inputted the outcome data into a predesigned spreadsheet. Any disagreements in the crosschecking process were resolved through discussion. Otherwise, a third investigator (LO) arbitrated the dispute. The main information extracted from the included articles included study design, populations studied, type of operation, type of anesthesia, intervention, outcomes, and postoperative test time. The Cochrane risk of bias tool was used to assess the methodological quality and the risks of bias of the individual studies (21). The certainty of the evidence for each outcome was 
evaluated using the grading of recommendations, assessment, development, and evaluations framework (22).

\section{Data Synthesis and Analysis}

Data were synthesized using RevMan version 5.4. For dichotomous outcomes, such as the incidence of POCD, we calculated the odds ratio (OR) and 95\% CI. For continuous outcomes, such as cognitive function scores, we pooled the mean difference (MD) and 95\% CI. Statistical significance was set at $p<0.05$.

\section{Subgroup Analyses and Investigation of Heterogeneity}

When sufficient studies reported relevant characteristics, we performed subgroup analyses for postoperative test time, anesthesia method, type of operation, and acupoint combination. We also conducted subgroup analyses to explore the impact of small sample-sized studies by grouping their sample size by quarter (from quarter 1, which includes $25 \%$ of the smallest trials, to quarter 4 , which includes $25 \%$ of the largest trials) (23). The heterogeneity among the studies was assessed using Cochran $\mathrm{Q}$ tests ( $\chi^{2}$ tests for heterogeneity), and significant statistical heterogeneity was defined as a Q test with $p<0.10$ or $I^{2}>50 \%$. We used the random-effects model to calculate the effect size, allowing for differences between the studies (24).

\section{Publication Bias and Sensitivity Analyses}

We tested for publication bias when sufficient studies were available $(n \geq 10)$. Asymmetric funnel and Harbord tests were applied to assess for potential publication bias when OR was used as an effect estimate, and the results showed there was no substantial heterogeneity between the studies. Moreover, when MD was regarded as an effect estimate, we adopted an asymmetric funnel and Egger's test to assess for potential publication bias (25). We also used the trim-and-fill method to identify and correct funnel plot asymmetries caused by publication bias (26). We conducted a sensitivity analysis to explore the source of heterogeneity by removing 1 study in each turn, and to examine the stability of the main outcome by excluding poor-quality trials with high risks of bias.

\section{RESULTS}

\section{Study Selection and Characteristics}

The database search identified a total of 371 articles. According to the inclusion and exclusion criteria, 29 RCTs (27-55) with 1,994 participants were eligible for data extraction. A flow diagram of the screening of the trials is shown in Figure 1. In the present review, all trials were conducted in China and published in Chinese and English. The average age of the participants in 11 trails $(27,28,35,36,39,40,43,44,46,47,51)$ was $18-$ 65 years and was $>65$ years in the other studies. Of the 29 reported interventions, 3 trails $(28,32,50)$ were treated with a combination of TEAS and controlled hypotension, and 1 (52) received TEAS combined with dexmedetomidine. Of 29 RCTs, 2 trials $(27,39)$ reported patients who underwent craniocerebral surgery, and 2 other studies $(48,53)$ involved patients undergoing cardiac surgery. Participants from the remaining 25 trails underwent non-cardiac surgery. The time points for evaluation in the included studies ranged from 0 to 7 days after surgery (Table 1).

\section{Quality Assessments}

All included studies were described as randomized. Among them, 22 studies $(27-29,31,32,34,37-39,41-45,48-55)$ (76\%) were performed using a random number table, and 1 (47) adopted drawing lots. Four studies $(51-53,55)(13 \%)$ described the proper way to complete allocation concealment. Seventeen studies (27, $29-31,34-40,42,43,46-48,50)(57 \%)$ were at high risk of bias for failing to blind participants and personnel, whereas the others were at low risk of bias. Fourteen studies $(29,31,32,34-$ $38,40,45,49,51,53,55)(48 \%)$ were blinded to the outcome assessment. All studies (100\%) showed low risks for incomplete outcome data. In this review, most of the research protocols (93\%) were not available on record, and so selective reporting was difficult to judge. The methodological quality of the included trials is shown in Figure 2.

\section{Primary Outcomes \\ Incidence of POCD}

Twenty-three studies $(28-43,45,47,49,50,53-55)$ reported the incidence of POCD. Overall, the results of the meta-analysis suggested a significantly lower incidence of POCD in the TEAS group than that in the CG $[\mathrm{OR}=0.40$ (95\%CI: $0.33,0.48) ; p$ $\left.<0.001, I^{2}=0 \%\right]$. Subgroup analyses by evaluation time points (postoperative days 1, 3, 5, and 7) showed that the TEAS group had a significantly lower incidence of POCD compared with that in the CG on postoperative days 1 [OR $=0.33$ (95\%CI: $0.23,0.47$ ); $p<0.001, I^{2}=0 \%$, moderate certainty], 3 [OR $=0.38$ (95\%CI: $0.29,0.50) ; p<0.001, I^{2}=0 \%$, low certainty], and 7 [OR $=0.51$ (95\%CI: $0.32,0.81$ ); $p=0.005, I^{2}=0 \%$, low certainty]. On postoperative day 5, no significant difference was observed between the TEAS group and the CG for the incidence of POCD [OR $=0.70$ (95\%CI: 0.36, 1.36); $p=0.29, I^{2}=0 \%$, low certainty] (Figure 3 and Table 2). Subgroup analysis of the operation type showed that compared with the CG, TEAS significantly reduced the incidence of POCD in patients undergoing non-cardiac and noncraniocerebral surgery [OR $=0.40$ (95\%CI: $0.33,0.49) ; p<$ $0.001, I^{2}=0 \%$, moderate certainty]. There was no significant difference in the incidence of POCD between the TEAS group and the CG, either for patients receiving craniocerebral surgery [only 1 study (39) reported, OR $=0.32$ (95\%CI: 0.03, 3.18); $p=0.33, I^{2}$ not applicable, low certainty] or cardiac surgery [only 1 study (53) reported, $\mathrm{OR}=0.37$ (95\%CI: $0.14,0.99) ; p=0.05$, $I^{2}$ not applicable, moderate certainty]. Furthermore, a subgroup analysis based on the different anesthesia techniques suggested a significant reduction of POCD-related morbidity in the TEAS group, regardless of using intravenous anesthesia $[\mathrm{OR}=0.44$ (95\%CI: 0.34, 0.56); $p<0.001, I^{2}=0 \%$, low certainty], intravenous-inhalation anesthesia $[\mathrm{OR}=0.38$ (95\%CI: 0.27 , 0.52); $p<0.001, I^{2}=0 \%$, low certainty], or combined spinal epidural anesthesia (CSEA) [OR $=0.35$ (95\%CI: 0.19, 0.62); $p<$ $0.001, I^{2}=0 \%$, low certainty] (Supplementary Table 1). 


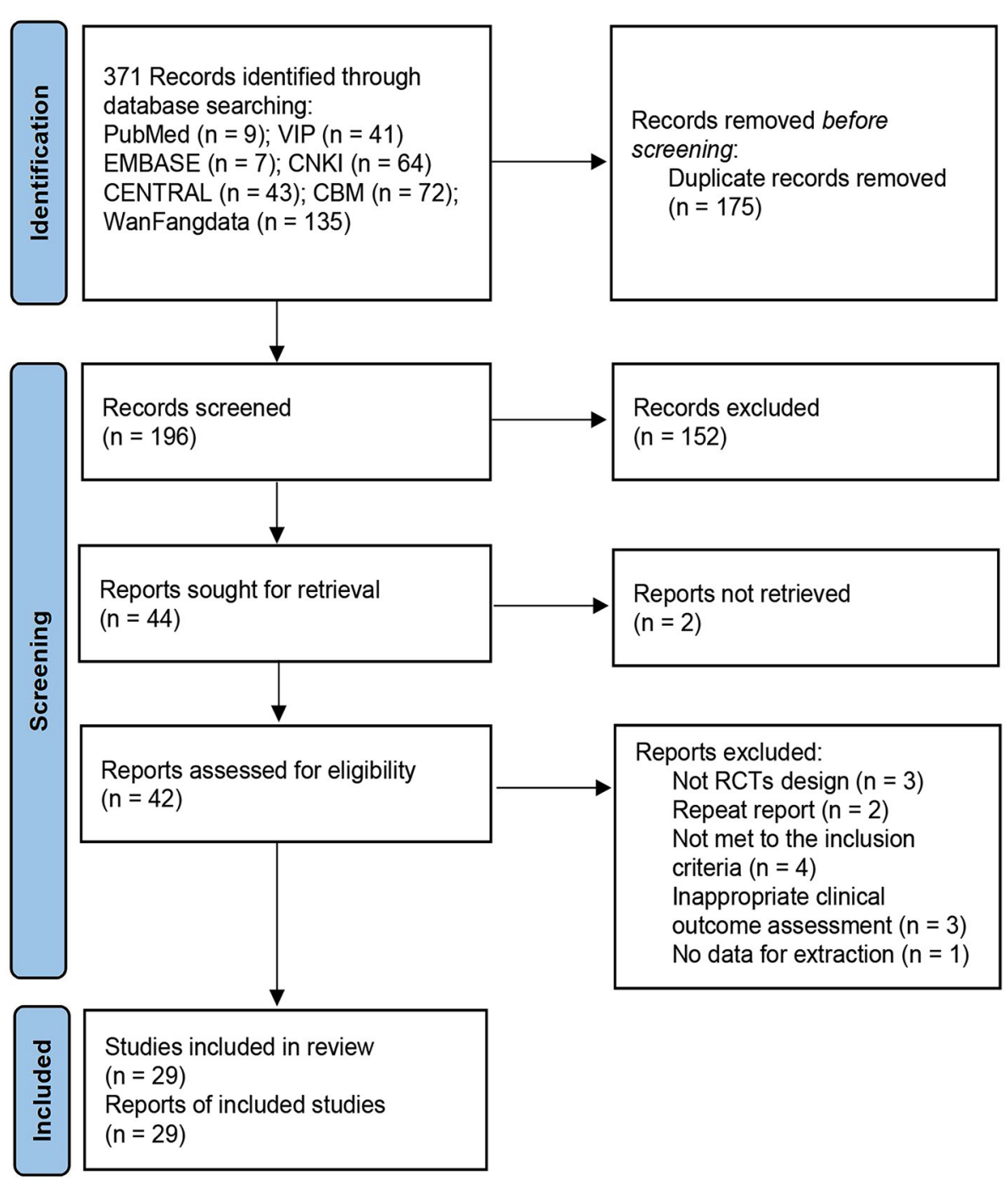

FIGURE 1 | Flowchart of study selection.

In the subgroup analysis of acupoint selection, we observed the efficacy of 6 kinds of acupoint combinations that were used more than 2 times in the included studies. Our metaanalysis showed that TEAS significantly reduced the incidence of POCD in 5 of the 6 combinations compared with CG. The acupoint combinations arranged in descending order of effect size were LI4, LI11, ST36, SP6 [OR = 0.33 (95\%CI: 0.14, 0.77); $p=0.01$, $I^{2}=0 \%$, low certainty]; GV20, PC6, ST36, SP6 [OR $=0.37$ (95\%CI: 0.26, 0.54); $p<0.001, I^{2}=0 \%$, low certainty]; GV20, PC6, GB20 [OR = 0.38 (95\%CI: 0.21, 0.71); $p=0.002, I^{2}=0 \%$, low certainty]; PC6, ST36 [OR $=0.40$ (95\%CI: 0.23, 0.69); $p$ $<0.001, I^{2}=0 \%$, low certainty]; and LI4, PC6 [OR $=0.49$ (95\%CI: 0.31, 0.77); $p=0.002, I^{2}=0 \%$, low certainty]. Nevertheless, acupoint combination containing GV20, PC6, and ST36 in the TEAS group had no advantage in preventing POCD [OR $=0.53$ (95\%CI: $0.27,1.04) ; p=0.07, I^{2}=0 \%$, low certainty] (Supplementary Table 1).
The funnel plot of the above analysis showed no significant asymmetry (Supplementary Figure 1); moreover, the Harbord test showed no publication bias $(p=0.647$; Supplementary Figures 2, 3). Additionally, the trim-andfill method revealed that publication bias had little effect on the combined results and that the results were stable (Supplementary Figures 4, 5). Further sensitivity analysis by excluding low-quality studies showed that the pooled analysis results were stable (Supplementary Table 2).

\section{Cognitive Function Score}

The included articles applied 5 types of cognitive function scoring methods (including MMSE, MoCA, and QoR-15/40) used in the included articles. We eventually selected MMSE scores $(n=24$ studies) to extract data for analysis $(27,29,31-38,40-42,44-$ $46,48-55)$. One study (51) reported data in a figure, which was excluded from this analysis because of the absence of 
TABLE 1 | Characteristics of the included randomized clinical trials.

\begin{tabular}{|c|c|c|c|c|c|c|c|c|}
\hline \multirow[t]{2}{*}{ First author } & \multirow{2}{*}{$\begin{array}{l}\text { Age (years) } \\
\text { EG/CG }\end{array}$} & \multirow{2}{*}{$\begin{array}{l}\text { Sample } \\
\text { size }\end{array}$} & \multirow{2}{*}{$\begin{array}{l}\text { Type of operation/ } \\
\text { anesthesia }\end{array}$} & \multicolumn{3}{|c|}{ Intervention } & \multirow[t]{2}{*}{ Outcomes } & \multirow{2}{*}{$\begin{array}{l}\text { Postoperative } \\
\text { test time }\end{array}$} \\
\hline & & & & EG & CG & $\begin{array}{l}\text { Acupoints/Stimulation } \\
\text { frequency/time }\end{array}$ & & \\
\hline Ren (27) & $\begin{array}{l}52 \pm 9 / \\
54 \pm 10\end{array}$ & 50 & $\begin{array}{l}\text { Craniotomy/ } \\
\text { Intravenous-inhalation }\end{array}$ & TEAS & No treatment & $\begin{array}{l}\mathrm{LI} 4 \text {, LI11, ST36, SP6; } 2 \sim 100 \mathrm{~Hz} \text {; } \\
\text { from } 30 \text { min before anesthesia to } \\
\text { the completion of operation }\end{array}$ & MMSE & $1,24,48 \mathrm{~h}$ \\
\hline Zhou et al. (28) & $45 \sim 65$ & 75 & $\begin{array}{l}\text { Single spinal } \\
\text { surgery/Intravenous-inhalation }\end{array}$ & $\begin{array}{l}\text { TEAS+ } \\
\text { Controlled } \\
\text { hypotension }\end{array}$ & Controlled hypotension & $\begin{array}{l}\mathrm{LI} 4, \mathrm{~L} 111, \mathrm{ST} 36, \mathrm{SP} 6 ; 2 \sim 100 \mathrm{~Hz} \text {; } \\
\text { from } 30 \text { min before anesthesia to } \\
\text { the completion of operation }\end{array}$ & $\begin{array}{l}\text { incidence of } \\
\text { POCD }\end{array}$ & $1,3,5 d$ \\
\hline Lin et al. (29) & $\begin{array}{l}68.5 \pm 2.8 / \\
67.3 \pm 2.7\end{array}$ & 49 & $\begin{array}{l}\text { Abdominal surgery/ } \\
\text { Intravenous }\end{array}$ & TEAS & No treatment & $\begin{array}{l}\text { GV20, GV29, PC6; 2 100 Hz; } \\
\text { from } 30 \text { min before anesthesia to } \\
\text { the completion of operation }\end{array}$ & $\begin{array}{l}\text { incidence of } \\
\text { POCD, MMSE }\end{array}$ & $3 d$ \\
\hline Wu et al. (30) & $62 \sim 76$ & 100 & $\begin{array}{l}\text { Laparoscopic cholecystectomy/ } \\
\text { Intravenous-inhalation }\end{array}$ & TEAS & No treatment & $\begin{array}{l}\text { GV20, PC6, ST36; } 2 \sim 15 \mathrm{~Hz} \text {; } \\
\text { from } 30 \text { min before anesthesia to } \\
\text { the completion of operation }\end{array}$ & $\begin{array}{l}\text { incidence of } \\
\text { POCD and PONV }\end{array}$ & $3,7 d$ \\
\hline Ni et al. (31) & $65 \sim 78$ & 60 & $\begin{array}{l}\text { Laparoscopic resection of rectal } \\
\text { cancer/Intravenous }\end{array}$ & TEAS & No treatment & $\begin{array}{l}\text { GV20, PC6, ST36, SP6; } \\
2 \sim 100 \mathrm{~Hz} \text {; from } 30 \text { min before } \\
\text { anesthesia to the completion of } \\
\text { operation }\end{array}$ & $\begin{array}{l}\text { incidence of } \\
\text { POCD, MMSE }\end{array}$ & $1,3,5,7 d$ \\
\hline Yin et al. (32) & $\begin{array}{l}78.3 \pm 5.5 / \\
77.5 \pm 5.2\end{array}$ & 53 & $\begin{array}{l}\text { Hip-replacement surgery/ } \\
\text { Intravenous }\end{array}$ & $\begin{array}{l}\text { TEAS+ } \\
\text { Controlled } \\
\text { hypotension }\end{array}$ & $\begin{array}{l}\text { Sham stimulation }+ \\
\text { Controlled hypotension }\end{array}$ & $\begin{array}{l}\text { GV20, PC6, GB20; 2 100 Hz; } \\
\text { from } 30 \text { min before anesthesia to } \\
\text { the completion of operation }\end{array}$ & $\begin{array}{l}\text { incidence of } \\
\text { POCD, MMSE }\end{array}$ & $72 \mathrm{~h}$ \\
\hline Yang et al. (33) & $65 \sim 80$ & 60 & $\begin{array}{l}\text { Gynecological laparoscopic } \\
\text { surgery/Intravenous }\end{array}$ & TEAS & Sham stimulation & $\begin{array}{l}\text { PC6, ST36; } 2 \sim 100 \mathrm{~Hz} \text {; from } \\
30 \text { min before anesthesia to the } \\
\text { completion of operation }\end{array}$ & $\begin{array}{l}\text { incidence of } \\
\text { POCD, MMSE }\end{array}$ & $1,3,5 d$ \\
\hline Wang(a) et al. (34) & $\begin{array}{l}69.9 \pm 4.2 / \\
69.3 \pm 4.1\end{array}$ & 60 & $\begin{array}{l}\text { Artificial femoral head } \\
\text { replacement/CSEA }\end{array}$ & TEAS & No treatment & $\begin{array}{l}\text { GV20, PC6, ST36, SP6; } \\
\text { 2 100 Hz; after anesthesia to } \\
\text { the end of operation }\end{array}$ & $\begin{array}{l}\text { incidence of } \\
\text { POCD, MMSE, } \\
\text { VAS }\end{array}$ & $1,3,7 d$ \\
\hline Zhu et al. (35) & $\begin{array}{l}34.2 \pm 9.7 / \\
34.6 \pm 8.4\end{array}$ & 60 & $\begin{array}{l}\text { Gynecological laparoscopic } \\
\text { surgery/Intravenous-inhalation }\end{array}$ & TEAS & No treatment & $\begin{array}{l}\text { PC6, ST36; 2 100 Hz; from } \\
30 \text { min before anesthesia to the } \\
\text { completion of operation }\end{array}$ & $\begin{array}{l}\text { incidence of } \\
\text { POCD, MMSE }\end{array}$ & $1 d$ \\
\hline Wei et al. (36) & $\begin{array}{l}55.2 \pm 6.1 / \\
56.5 \pm 4.6\end{array}$ & 40 & $\begin{array}{l}\text { Gynecological laparoscopic } \\
\text { surgery/Intravenous-inhalation }\end{array}$ & TEAS & No treatment & $\begin{array}{l}\text { GV20, PC6, GB2O; 2 100 Hz; } \\
30 \text { min before anesthesia }\end{array}$ & $\begin{array}{l}\text { incidence of } \\
\text { POCD, MMSE }\end{array}$ & $1,3 d$ \\
\hline Li et al. (37) & $\begin{array}{l}65.7 \pm 6.1 / \\
66.5 \pm 4.0\end{array}$ & 60 & $\begin{array}{l}\text { Radical thoracoscopic lung } \\
\text { cancer operation/ } \\
\text { Intravenous-inhalation }\end{array}$ & TEAS & No treatment & $\begin{array}{l}\text { PC6, ST36; } 2 \sim 100 \mathrm{~Hz} \text {; from } \\
30 \text { min before anesthesia to the } \\
\text { completion of operation }\end{array}$ & $\begin{array}{l}\text { incidence of } \\
\text { POCD, MMSE }\end{array}$ & $1,3 d$ \\
\hline Wang(b) et al. (38) & $\begin{array}{l}70.3 \pm 4.2 / \\
69.5 \pm 4.4\end{array}$ & 60 & $\begin{array}{l}\text { Laparoscopic radical } \\
\text { gastrectomy for cancer/ } \\
\text { Intravenous }\end{array}$ & TEAS & No treatment & $\begin{array}{l}\text { GV20, PC6, ST36, SP6; } \\
2 \sim 100 \mathrm{~Hz} \text {; after anesthesia to } \\
\text { the end of operation }\end{array}$ & $\begin{array}{l}\text { incidence of } \\
\text { POCD, MMSE, } \\
\text { VAS }\end{array}$ & $1,3,7 d$ \\
\hline Zhao et al. (39) & $\begin{array}{l}37.0 \pm 11.7 / \\
37.9 \pm 11.9\end{array}$ & 80 & $\begin{array}{l}\text { Transsphenoidal surgery/ } \\
\text { Intravenous-inhalation }\end{array}$ & TEAS & No treatment & $\begin{array}{l}\mathrm{LI} 4 \text {, TE5, EX-HN4; } 2 \sim 100 \mathrm{~Hz} \text {; } \\
\text { from } 30 \text { min before anesthesia to } \\
\text { the completion of operation }\end{array}$ & $\begin{array}{l}\text { incidence of } \\
\text { POCD, } \\
\text { neuropsychological } \\
\text { tests }\end{array}$ & $3 d$ \\
\hline
\end{tabular}


TABLE 1 | Continued

\begin{tabular}{|c|c|c|c|c|c|c|c|c|}
\hline \multirow[t]{2}{*}{ First author } & \multirow{2}{*}{$\begin{array}{l}\text { Age (years) } \\
\text { EG/CG }\end{array}$} & \multirow{2}{*}{$\begin{array}{l}\text { Sample } \\
\text { size }\end{array}$} & \multirow{2}{*}{$\begin{array}{l}\text { Type of operation/ } \\
\text { anesthesia }\end{array}$} & \multicolumn{3}{|c|}{ Intervention } & \multirow[t]{2}{*}{ Outcomes } & \multirow{2}{*}{$\begin{array}{l}\text { Postoperative } \\
\text { test time }\end{array}$} \\
\hline & & & & EG & CG & $\begin{array}{l}\text { Acupoints/Stimulation } \\
\text { frequency/time }\end{array}$ & & \\
\hline Wei et al. (40) & $\begin{array}{l}57.9 \pm 3.9 / \\
57.6 \pm 4.1\end{array}$ & 40 & $\begin{array}{l}\text { Gynecological laparoscopic } \\
\text { surgery/ } \\
\text { Intravenous-inhalation }\end{array}$ & TEAS & No treatment & $\begin{array}{l}\text { GV20, PC6, GB20; 2 100 Hz; } \\
30 \text { min before anesthesia, } \\
\text { stimulation for } 1 \mathrm{~h}\end{array}$ & $\begin{array}{l}\text { incidence of } \\
\text { POCD, MMSE }\end{array}$ & $1,3 d$ \\
\hline Tan et al. (41) & $\begin{array}{l}67.1 \pm 6.2 / \\
66.4 \pm 5.5\end{array}$ & 70 & $\begin{array}{l}\text { Laparoscopic cholecystectomy/ } \\
\text { NR }\end{array}$ & TEAS & Sham stimulation & $\begin{array}{l}\text { GV20, PC6, ST36, SP6; } \\
2 \sim 100 \mathrm{~Hz} ; 30 \mathrm{~min} / \mathrm{d} \text { from the } \\
\text { first day before operation to the } 7 \\
\text { day after operation }\end{array}$ & $\begin{array}{l}\text { incidence of } \\
\text { POCD, MMSE }\end{array}$ & $3,7 d$ \\
\hline Tang et al. (42) & $\begin{array}{l}69.6 \pm 5.8 / \\
70.1 \pm 6.3\end{array}$ & 90 & $\begin{array}{l}\text { Colorectal cancer surgery/ } \\
\text { intravenous }\end{array}$ & TEAS & No treatment & $\begin{array}{l}\text { GV20, GV24; } 2 \sim 100 \mathrm{~Hz} \text {; from } \\
30 \text { min before anesthesia to the } \\
\text { completion of operation }\end{array}$ & $\begin{array}{l}\text { incidence of } \\
\text { POCD, MMSE }\end{array}$ & $1,3,5,7 d$ \\
\hline Fan et al. (43) & $\begin{array}{l}54 \pm 7 / \\
54 \pm 8\end{array}$ & 56 & $\begin{array}{l}\text { Laparoscopic resection of } \\
\text { colorectal cancer/ } \\
\text { Intravenous-inhalation }\end{array}$ & TEAS & No treatment & $\begin{array}{l}\text { PC6, LI4, ST36, ST37, ST39; } \\
2 \sim 100 \mathrm{~Hz} \text {; from } 30 \text { min before } \\
\text { anesthesia to the completion of } \\
\text { operation }\end{array}$ & $\begin{array}{l}\text { incidence of } \\
\text { POCD and PONV, } \\
\text { QoR-15, length of } \\
\text { hospital stay }\end{array}$ & $3 d$ \\
\hline Mi et al. (44) & $\begin{array}{l}44 \pm 6 / \\
45 \pm 8\end{array}$ & 100 & $\begin{array}{l}\text { Laparoscopic cholecystectomy/ } \\
\text { Intravenous-inhalation }\end{array}$ & TEAS & Sham stimulation & $\begin{array}{l}\text { LI4, PC6, ST36; 2 100 Hz; from } \\
30 \text { min before anesthesia to the } \\
\text { completion of operation }\end{array}$ & $\begin{array}{l}\text { QoR-40, MMSE, } \\
\text { incidence of PONV }\end{array}$ & $4,8,24,48 \mathrm{~h}$ \\
\hline Sun et al. (45) & $\begin{array}{l}86.3 \pm 4.4 / \\
85.8 \pm 4.2\end{array}$ & 40 & Hip fracture surgery/CSEA & TEAS & Sham stimulation & $\begin{array}{l}\text { GV2O, GB2O; 2 100 Hz; (3 } \\
\text { times/d, } 30 \text { min/time) since } 2 \\
\text { days before operation until the } \\
\text { operation finished }\end{array}$ & $\begin{array}{l}\text { incidence of } \\
\text { POCD, MMSE }\end{array}$ & $24,72 \mathrm{~h}$ \\
\hline Mao et al. (46) & $35 \sim 55$ & 80 & $\begin{array}{l}\text { Breast cancer surgery/ } \\
\text { Intravenous }\end{array}$ & TEAS & No treatment & $\begin{array}{l}\text { LI4, PC6; 2 100 Hz; from } 10 \mathrm{~min} \\
\text { before anesthesia to the } \\
\text { completion of operation }\end{array}$ & $\begin{array}{l}\text { MMSE, incidence } \\
\text { of PONV }\end{array}$ & $24,48 \mathrm{~h}$ \\
\hline Li and Yang (47) & $\begin{array}{l}39.0 \pm 5.2 / \\
39.0 \pm 5.3\end{array}$ & 90 & $\begin{array}{l}\text { Laparoscopic Myomectomy/ } \\
\text { Intravenous-inhalation }\end{array}$ & TEAS & Dex & $\begin{array}{l}\text { PC6, ST36; 2 100 Hz; from } \\
30 \text { min before anesthesia to the } \\
\text { completion of operation }\end{array}$ & $\begin{array}{l}\text { incidence of } \\
\text { POCD and PONV }\end{array}$ & $3 d$ \\
\hline Wu and Chen (48) & $\begin{array}{l}72.3 \pm 5.3 / \\
71.9 \pm 5.1\end{array}$ & 84 & $\begin{array}{l}\text { Cardiac surgery/ } \\
\text { Intravenous }\end{array}$ & TEAS & No treatment & $\begin{array}{l}\text { GV20, PC6, ST36, SP6; } \\
\text { 2 100 Hz; from } 30 \text { min before } \\
\text { anesthesia to the completion of } \\
\text { operation }\end{array}$ & $\begin{array}{l}\text { MMSE, VAS, } \\
\text { incidence of PONV }\end{array}$ & $3 d$ \\
\hline Duan et al. (49) & $\begin{array}{l}78 \pm 10 / \\
76 \pm 11\end{array}$ & 80 & $\begin{array}{l}\text { Hip replacement/ } \\
\text { Intravenous-inhalation }\end{array}$ & TEAS & Sham stimulation & $\begin{array}{l}\text { LI4, PC6; 2 200 Hz; from } 30 \mathrm{~min} \\
\text { before anesthesia to the } \\
\text { completion of operation }\end{array}$ & $\begin{array}{l}\text { incidence of } \\
\text { POCD, MMSE, } \\
\text { VAS }\end{array}$ & $24,72 \mathrm{~h}$ \\
\hline Lu et al. (50) & $\begin{array}{l}72.1 \pm 2.5 / \\
71.3 \pm 2.3\end{array}$ & 91 & $\begin{array}{l}\text { Hip replacement/ } \\
\text { Intravenous-inhalation }\end{array}$ & $\begin{array}{l}\text { TEAS+ } \\
\text { Controlled } \\
\text { hypotension }\end{array}$ & Controlled hypotension & $\begin{array}{l}\text { GV20, PC6, GB20; 2 100 Hz; } \\
\text { Before anesthesia induction to } \\
\text { the end of operation }\end{array}$ & $\begin{array}{l}\text { incidence of } \\
\text { POCD and PONV, } \\
\text { MMSE, VAS }\end{array}$ & $72 \mathrm{~h}$ \\
\hline Yu et al. (51) & $\begin{array}{l}48.5 \pm 16.2 / \\
45.9 \pm 17.5\end{array}$ & 60 & $\begin{array}{l}\text { Gynecological laparoscopic } \\
\text { surgery/ } \\
\text { Intravenous }\end{array}$ & TEAS & Sham stimulation & $\begin{array}{l}\text { GV20, GV29, ST36, PC6; } \\
\text { 2 100 Hz; } 30 \text { min before } \\
\text { anesthesia }\end{array}$ & $\begin{array}{l}\text { QoR-40, MMSE, } \\
\text { VAS, incidence of } \\
\text { PONV }\end{array}$ & $1,2 d$ \\
\hline
\end{tabular}




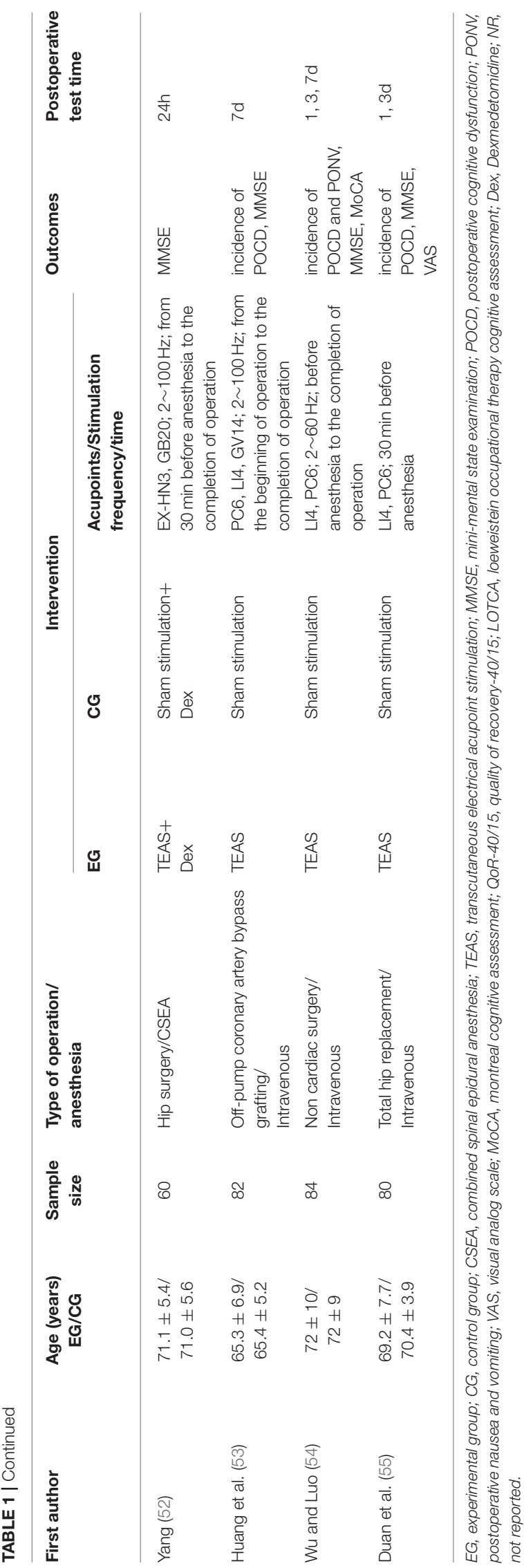

available data. The meta-analysis of different time points after surgery suggested that, compared with the CG, TEAS resulted in significantly improved MMSE scores on postoperative day $1\left[\mathrm{MD}=2.44\right.$ (95\%CI: 1.61, 3.27); $p<0.001, I^{2}=93 \%$, low certainty], $3\left[\mathrm{MD}=2.07\right.$ (95\%CI: 1.53, 2.62); $p<0.001, I^{2}=87 \%$, low certainty], and 7 [MD $0.49=(95 \%$ CI: $0.18,0.79) ; p=0.002$, $I^{2}=21 \%$, low certainty] but not on day $5[\mathrm{MD}=0.98(95 \% \mathrm{CI}$ : $-0.03,1.99$ ); $P=0.06, I^{2}=70 \%$, very low certainty] (Figure 4 and Table 2).

Based on the above analysis, visual inspection showed an asymmetrical funnel plot (Supplementary Figure 6); however, Egger's test did not detect publication bias $(p=0.20$; Supplementary Figures 7, 8). Therefore, we used the trimand-fill method for further sensitivity analyses. After including the estimated missing studies, the imputed studies produced a symmetrical funnel plot, and the results after trimming and filling showed a significantly higher MMSE score in the TEAS group than that in the CG $[\mathrm{MD}=1.48$ (95\%CI: 1.03, 1.93)] (Supplementary Figures 9, 10). Considering the substantial heterogeneity, further sensitivity analysis by excluding individual studies or low-quality studies suggested that the pooled analysis results were stable and no significant source of heterogeneity was found (Supplementary Table 2, Supplementary Figures 11-14).

\section{Secondary Outcomes}

Seven studies (34, 38, 48-51, 55) reported postoperative visual analog scale scores. The results of the meta-analysis demonstrated that TEAS had a significantly greater pain reduction compared with the $\mathrm{CG}$ at $8 \mathrm{~h}[\mathrm{MD}=-0.39(95 \% \mathrm{CI}$ : $-0.73,-0.05) ; p=0.03, I^{2}=64 \%$, low certainty], $12 \mathrm{~h}$ $[\mathrm{MD}=-0.31$ (95\%CI: $-0.43,-0.20) ; p<0.001, I^{2}=0 \%$, moderate certainty], and $24 \mathrm{~h}$ [MD $=-0.46$ (95\%CI: -0.74 , $-0.17) ; p=0.002, I^{2}=85 \%$, low certainty] postoperatively, but not at $48 \mathrm{~h}[\mathrm{MD}=-0.36$ (95\%CI: $-0.81,0.10) ; p=0.12$, $I^{2}=84 \%$, low certainty] (Figure 5 and Table 2). Considering the significant heterogeneity between studies, we changed the pooled effect index to SMD. The heterogeneity was significantly reduced, and the pooled results remains statistically significant for $24 \mathrm{~h}$ after surgery (Supplementary Figure 15). Nine studies reported the incidence of PONV $(30,43,44,46-48,50,51,54)$. Metaanalysis showed a significantly lower incidence of PONV in the TEAS group than in the CG [OR $=0.36$ (95\%CI: $0.22,0.58$ ); $p$ $<0.001, I^{2}=0 \%$, low certainty] (Figure 6 and Table 2). Only 1 trial (43) was included in the meta-analysis of the effect of TEAS on the length of hospital stay. As shown in Figure 7 and Table 2, compared with the CG, the length of hospital stay in the TEAS group was significantly shorter $[\mathrm{MD}=-2.50$ (95\%CI: -3.91 , $-1.09) ; p<0.001$, low certainty].

\section{DISCUSSION}

Transcutaneous electrical acupoint stimulation has been widely used in clinical practice and has become an important part of perioperative management. TEAS provides many benefits in promoting rehabilitation after surgery from many aspects, 
Random sequence generation (selection bias)

Allocation concealment (selection bias)

Blinding of participants and personnel (performance bias)

Blinding of outcome assessment (detection bias)

Incomplete outcome data (attrition bias)

Selective reporting (reporting bias)

Other bias

Low risk of bias

$$
\text { Other bias }
$$

\begin{tabular}{|ll|}
\hline Low risk of bias $\quad \square$ Unclear risk of bias $\quad \square$ High risk of bias \\
\hline
\end{tabular}

\begin{tabular}{|c|c|c|c|c|c|c|c|c|c|c|c|c|c|c|c|c|c|c|c|c|c|c|c|c|c|c|c|c|}
\hline 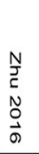 & & & & 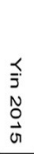 & 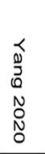 & & & 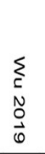 & & $\begin{array}{l}\frac{k}{0} \\
\underline{0} \\
N \\
0 \\
\vec{v}\end{array}$ & 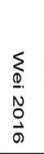 & & & & $\begin{array}{l}-1 \\
\tilde{u} \\
\tilde{N} \\
\tilde{u} \\
\vec{v}\end{array}$ & & 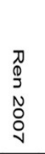 & & & & 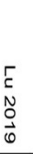 & & & $\begin{array}{l}\frac{5}{N} \\
\stackrel{0}{a} \\
\vec{\sigma}\end{array}$ & & & 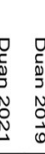 & \\
\hline$\nu$ & + & + & + & + & + & $\nu$ & 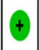 & + & $\nu$ & $\nu$ & $\nu$ & + & $\uparrow$ & + & + & 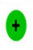 & + & + & + & $\nu$ & + & + & + & + & + & + & +4 & Random sequence generation (selection bias) \\
\hline$\nu$ & $\nu$ & $\nu$ & + & $\nu$ & + & $\nu$ & $\nu$ & $\nu$ & $\nu$ & 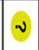 & $\nu$ & $\nu$ & $\nu$ & $\nu$ & $\nu$ & $\omega$ & $\nu$ & $\nu$ & $\nu$ & $\nu$ & $\nu$ & $\nu$ & $\nu$ & $\nu$ & + & $\nu$ & +2 & Allocation concealment (selection bias) \\
\hline 0 & + & 0 & + & + & + & + & + & 0 & 0 & 0 & 0 & 0 & 0 & 0 & + & + & 0 & 0 & + & 0 & 0 & 0 & 0 & 0 & + & 0 & $+t$ & Blinding of participants and personnel (performance bias) \\
\hline+ & $\nu$ & $\nu$ & + & + & $\nu$ & $\nu$ & $\nu$ & $\nu$ & $\nu$ & + & + & + & $\uparrow$ & $\nu$ & $\nu$ & + & $\nu$ & + & $\nu$ & $\nu$ & $\nu$ & + & $\nu$ & + & + & $\nu$ & +4 & Blinding of outcome assessment (detection bias) \\
\hline+ & + & + & + & + & + & + & + & + & + & + & + & + & $\uparrow$ & + & + & + & + & + & + & + & $\uparrow$ & + & + & 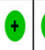 & + & + & $+t$ & Incomplete outcome data (attrition bias) \\
\hline$\nu$ & $\nu$ & $\nu$ & $\uparrow$ & $\nu$ & $\nu$ & $\nu$ & $\nu$ & $\nu$ & $\nu$ & $\nu$ & $\nu$ & $\nu$ & $\nu$ & $\nu$ & $\nu$ & $\nu$ & $\nu$ & $\nu$ & $\nu$ & $\nu$ & $\nu$ & $\nu$ & $\nu$ & $\nu$ & + & $\nu$. & $\nu$ & Selective reporting (reporting bias) \\
\hline$\uparrow$ & + & + & + & + & + & + & + & + & + & + & + & $\uparrow$ & + & + & + & 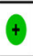 & + & + & + & 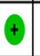 & + & + & + & 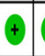 & + & + & + & Other bias \\
\hline
\end{tabular}

FIGURE 2 | Risk of bias graph.

including analgesia, gastrointestinal tract regulation, antiinflammatory effects, stress response reduction, and POCD prevention (56). In this study, we examined the preventive effect of TEAS on postoperative short-term cognitive change. Our results showed that TEAS exerted a significant effect on increasing MMSE scores and lowering the incidence of POCD on postoperative days 1,3 , and 7 . On a postoperative day 5, TEAS showed no significant therapeutic advantage in the primary outcomes, namely the incidence of POCD and MMSE scores. As reported in the literature, POCD, as a syndrome of prolonged impairment of cognitive function, occurs immediately to several years after surgery and usually persists for weeks or months (57). The effects of TEAS on medium- and longterm postoperative follow-up are still unclear. Anesthesia and surgery may be involved in the incidence of POCD. A previous meta-analysis showed that general anesthesia, compared to other forms, might increase the risk of developing POCD (58). A Cochrane systematic review suggested that, for elderly people undergoing non-cardiac surgery, maintenance with propofolbased total intravenous anesthesia might reduce the prevalence of POCD compared to maintenance with inhalational anesthesia (59). However, the effects of anesthesia and surgery on POCD remain controversial. A multicenter prospective cohort study indicated that exposure to surgery and general anesthesia were not significant risk factors for long-term cognitive impairment after major non-cardiac surgery associated with a critical illness (60). In this study, the subgroup analysis of anesthesia type showed that TEAS, compared with the CG, had a significant tendency toward a lower incidence of POCD, whether under general anesthesia (including intravenous and intravenousinhalation) or non-general anesthesia. Furthermore, we found significantly reduced POCD in patients who underwent noncardiac and noncraniocerebral surgery with TEAS. Of note, only one study involving cardiac surgery was included in this outcome. Based on the currently available data, there is insufficient evidence to recommend the use of TEAS in patients undergoing cardiac surgery.

The perioperative period is often accompanied by varying degrees of pain, which affects patient sleep, reduces the quality of life, and increases the length of hospital stay. TEAS do not only reduces pain and the incidence of complications caused by analgesics but also lessens the amount of anesthesia used in perioperative pain management (61). For postoperative pain, we found that TEAS enhanced the postoperative analgesic effect 


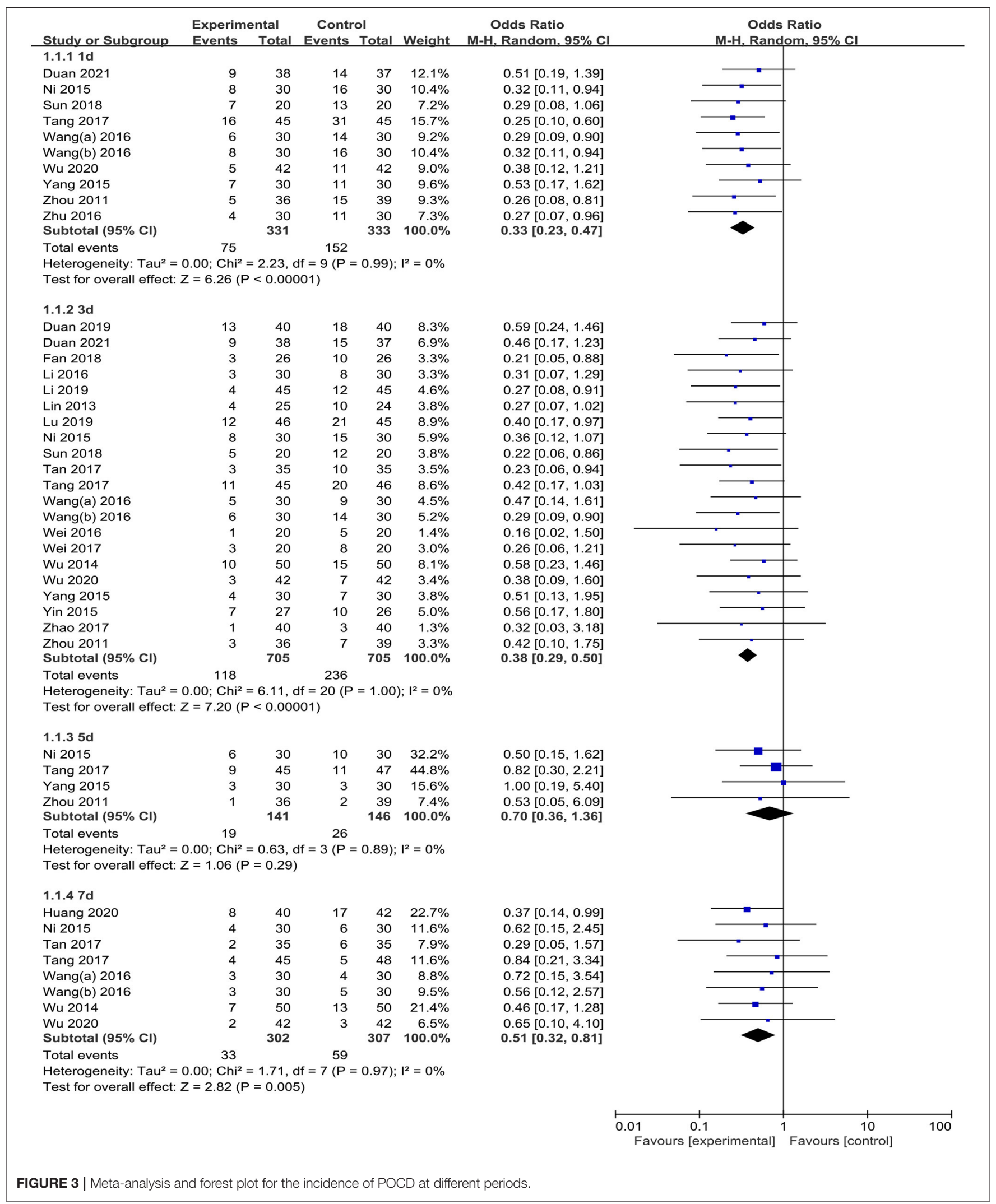


TABLE 2 | Main findings and evidence quality of the meta-analysis of TEAS for the prevention of POCD.

\begin{tabular}{|c|c|c|c|c|c|c|c|c|c|}
\hline \multirow[t]{2}{*}{ Outcomes } & \multirow{2}{*}{$\begin{array}{l}\text { No of studies } \\
\text { (Participants) }\end{array}$} & \multicolumn{6}{|c|}{ Quality assessment } & \multirow[t]{2}{*}{ Effect $(95 \% \mathrm{Cl})$} & \multirow[t]{2}{*}{ Quality } \\
\hline & & Design & Risk of bias & Inconsistency & Indirectness & Imprecision & Other considerations & & \\
\hline \multicolumn{10}{|l|}{ Incidence of POCD } \\
\hline $1 d$ & 10 studies (664) & $\mathrm{RCT}$ & Downgraded $^{a}$ & Not downgraded & Not downgraded & Not downgraded & Not downgraded & OR $0.33(0.23,0.47)$ & moderate \\
\hline $3 d$ & $\begin{array}{l}21 \text { studies } \\
(1410)\end{array}$ & $\mathrm{RCT}$ & Downgraded ${ }^{a}$ & Not downgraded & Not downgraded & Not downgraded & Downgraded $^{d}$ & OR $0.38(0.29,0.50)$ & low \\
\hline $5 d$ & 4 studies (287) & $\mathrm{RCT}$ & Downgradeda & Not downgraded & Not downgraded & Downgraded ${ }^{c}$ & Not downgraded & OR $0.70(0.36,1.36)$ & low \\
\hline $7 \mathrm{~d}$ & 8 studies (609) & $\mathrm{RCT}$ & Downgraded $^{a}$ & Not downgraded & Not downgraded & Downgraded ${ }^{C}$ & Not downgraded & OR $0.51(0.32,0.81)$ & low \\
\hline \multicolumn{10}{|l|}{ MMSE scores } \\
\hline $1 d$ & $\begin{array}{l}17 \text { studies } \\
\text { (1099) }\end{array}$ & RCT & Downgraded $^{a}$ & Downgraded ${ }^{b}$ & Not downgraded & Not downgraded & Not downgraded & MD 2.44 (1.61, 3.27) & low \\
\hline $3 d$ & $\begin{array}{l}17 \text { studies } \\
\text { (1096) }\end{array}$ & $\mathrm{RCT}$ & Downgradeda & Downgraded ${ }^{b}$ & Not downgraded & Not downgraded & Not downgraded & MD $2.07(1.53,2.62)$ & low \\
\hline $5 d$ & 3 studies (210) & $\mathrm{RCT}$ & Downgraded $^{a}$ & Downgraded ${ }^{b}$ & Not downgraded & Downgraded ${ }^{c}$ & Not downgraded & MD $0.98(-0.03,1.99)$ & very low \\
\hline $7 d$ & 7 studies (506) & $\mathrm{RCT}$ & Downgraded $^{a}$ & Not downgraded & Not downgraded & Not downgraded & Downgraded ${ }^{d}$ & MD $0.49(0.18,0.79)$ & low \\
\hline \multicolumn{10}{|l|}{ VAS scores } \\
\hline $8 \mathrm{~h}$ & 2 studies (175) & $\mathrm{RCT}$ & Downgradeda & Downgraded & Not downgraded & Not downgraded & Not downgraded & MD-0.39 $(-0.73,-0.05)$ & low \\
\hline $12 \mathrm{~h}$ & 2 studies (164) & $\mathrm{RCT}$ & Downgraded $^{a}$ & Not downgraded & Not downgraded & Not downgraded & Not downgraded & MD-0.31 $(-0.43,-0.20)$ & moderate \\
\hline $24 \mathrm{~h}$ & 7 studies (510) & $\mathrm{RCT}$ & Downgraded ${ }^{a}$ & Downgraded & Not downgraded & Not downgraded & Not downgraded & MD-0.46 $(-0.74,-0.17)$ & low \\
\hline $48 \mathrm{~h}$ & 3 studies (226) & $\mathrm{RCT}$ & Downgraded ${ }^{a}$ & Downgraded & Not downgraded & Not downgraded & Not downgraded & MD-0.36 $(-0.81,0.10)$ & low \\
\hline Incidence of PONV & 9 studies (741) & $\mathrm{RCT}$ & Downgraded ${ }^{a}$ & Not downgraded & Not downgraded & Downgraded ${ }^{C}$ & Not downgraded & OR $0.36(0.22,0.58)$ & low \\
\hline Length of hospital stay & 1 study (52) & $\mathrm{RCT}$ & Downgraded $^{a}$ & Not downgraded & Not downgraded & Downgraded ${ }^{c}$ & Not downgraded & MD-2.50 $(-3.91,-1.09)$ & low \\
\hline
\end{tabular}

TEAS, transcutaneous electrical acupoint stimulation; POCD, postoperative cognitive dysfunction; MMSE, mini-mental state examination; PONV, postoperative nausea and vomiting; VAS, visual analog scale; RCT, randomized controlled trial; $\mathrm{Cl}$, confidence intervals; $\mathrm{OR}$, odds ratio; $M D$, mean difference.

${ }^{a}$ Downgraded by 1 level because unclear risk of bias was likely to lower confidence in the estimate of effect.

${ }^{b}$ Downgraded by 1 level because heterogeneity $\left(l^{2}>50 \%\right)$.

${ }^{c}$ Downgraded by 1 level because total (cumulative) sample size was lower than the calculated optimal information size (O/S) and/or 95\% Cl included a null effect.

${ }^{d}$ Downgraded by 1 level because reporting bias $(p<0.1)$. 


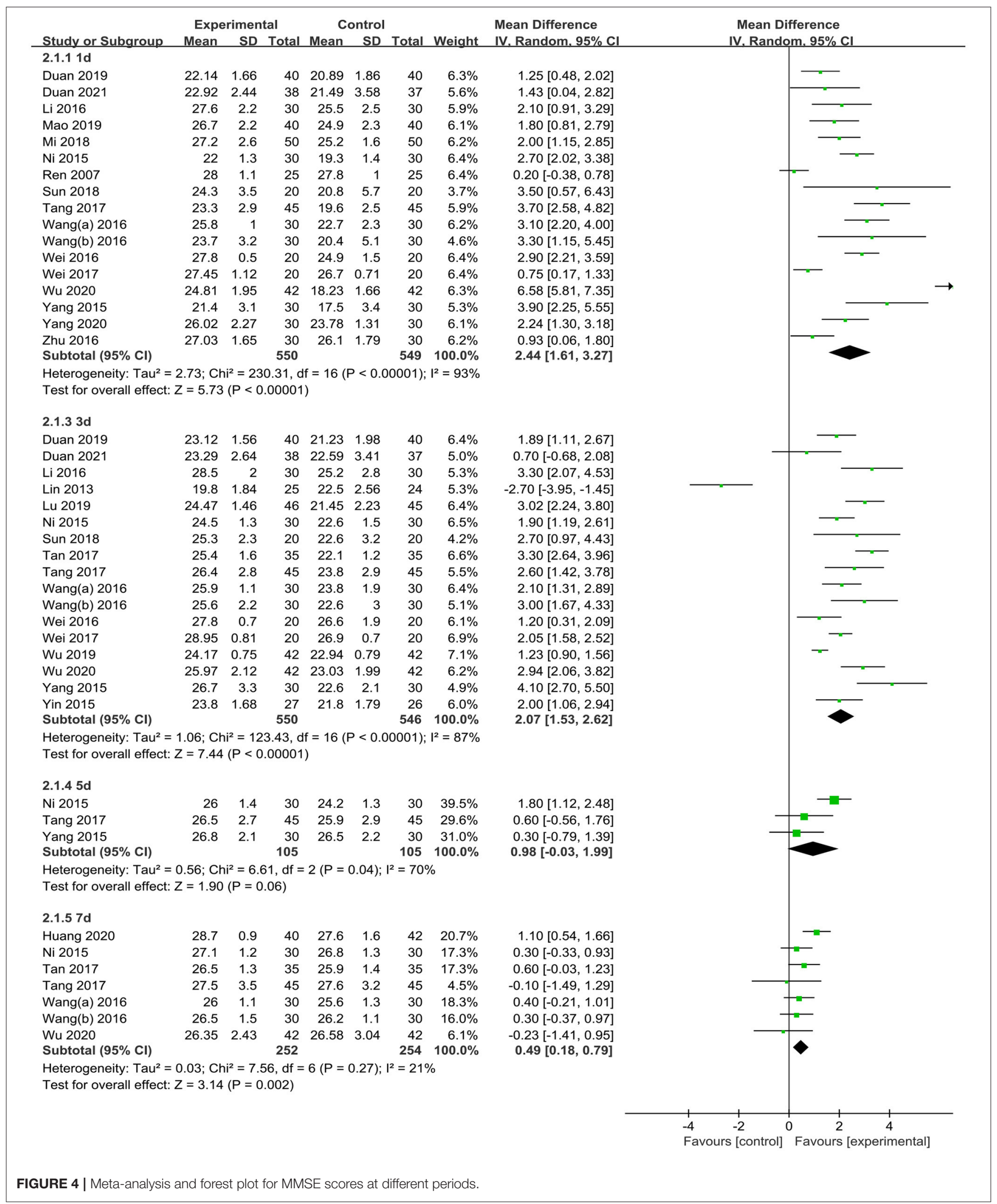




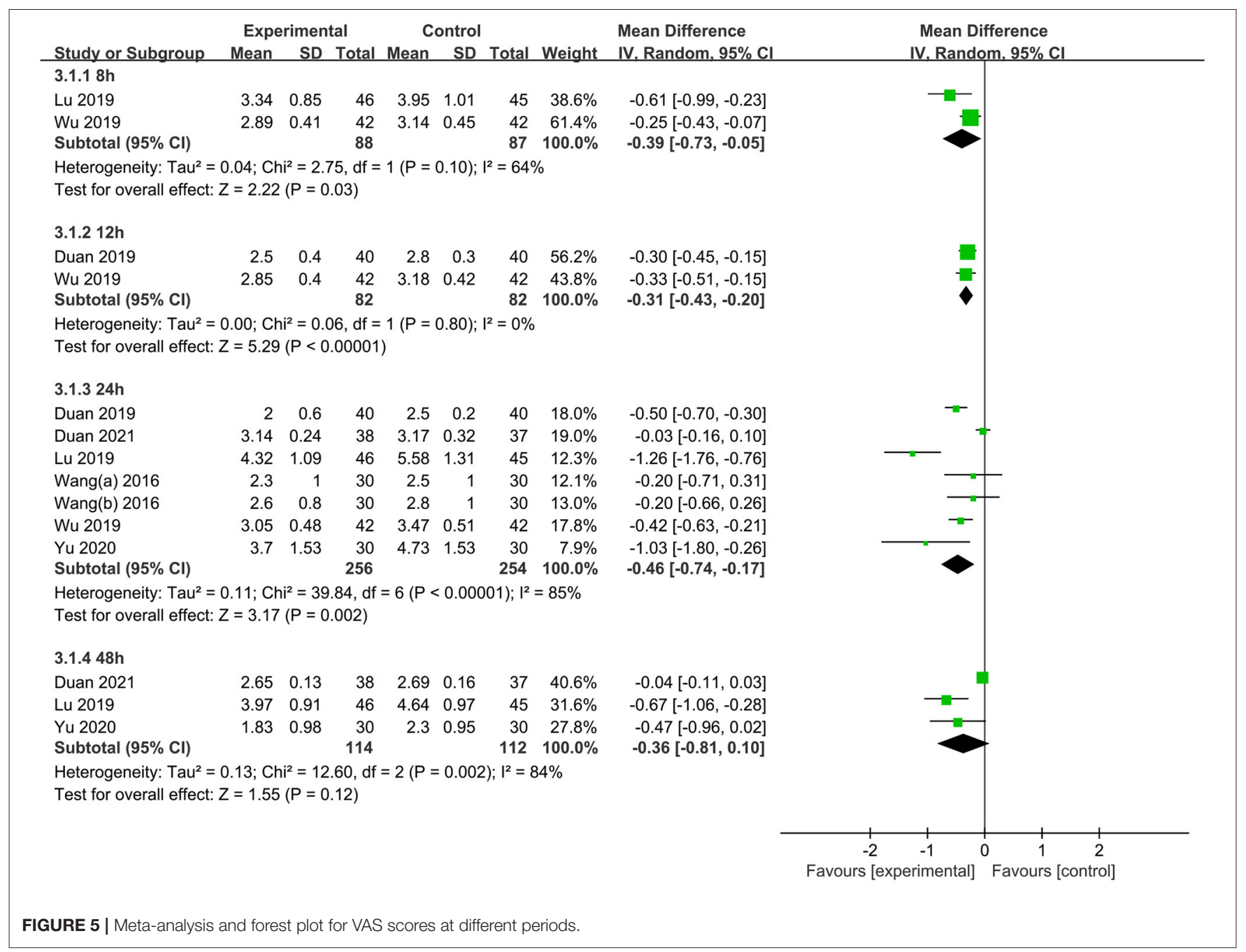

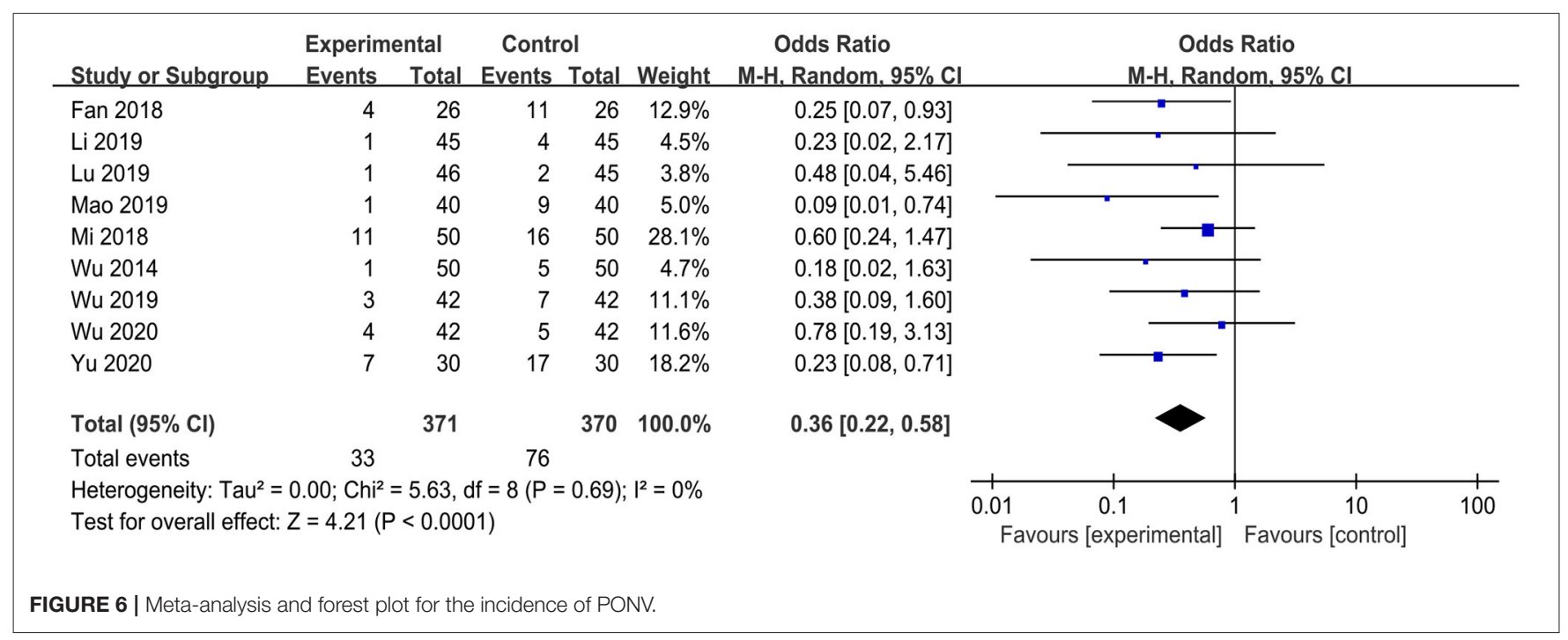




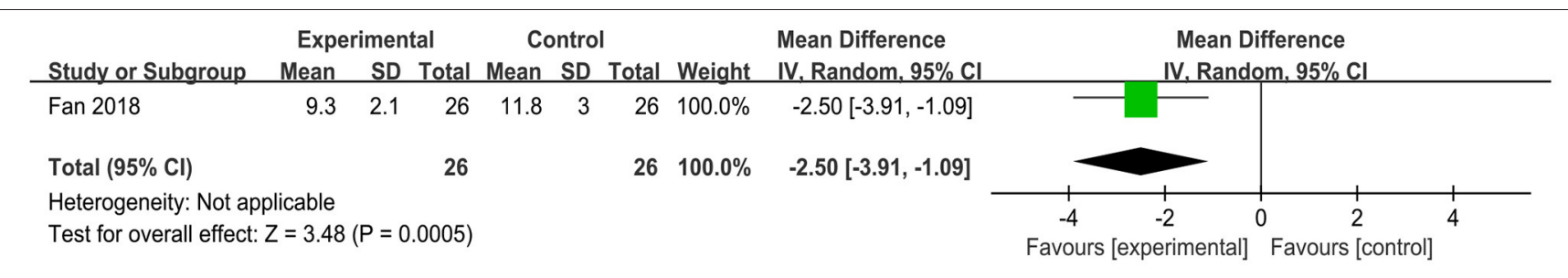

FIGURE 7 | Meta-analysis and forest plot for the length of hospital stay.

within $24 \mathrm{~h}$ after surgery. Furthermore, TEAS also effectively promoted the recovery of gastrointestinal function after surgery by increasing the level of plasma excitatory gastrointestinal hormone (62). A previous meta-analysis including 14 RCTs suggested that TEAS showed evidence of PONV prevention after general anesthesia (63), which was consistent with our findings. In general, POCD is associated with an increased length of hospital stay. Our review found that TEAS had advantages in shortening the postoperative hospital stay. Moreover, the selection of acupoints is an important factor in the TEAS treatment. The top 3 acupoints used in the included studies were PC6 (23 times), ST36 (15 times), and GV20 (14 times), which have the function of Kaiqiao Xingnao according to the theory of traditional Chinese medicine. As for the choice of acupoint combinations, we believe that the best combination is LI4, LI11, ST36, and SP6.

Transcutaneous electrical acupoint stimulation is "acupuncture-like TENS," which is an ideal combination of acupoints and bioelectricity. Compared with traditional acupuncture or electroacupuncture, TEAS is a non-invasive therapy. The potential mechanism of TEAS in preventing POCD may be explained by the following: first, TEAS alleviates postoperative inflammatory injury and reduces cytokine interleukin- $1 \beta$ (IL-1 $\beta$ ) and tumor necrosis factor- $\alpha$ (TNF- $\alpha$ ) levels in the central nervous system and peripheral circulation $(32,49,53,64)$; second, TEAS can reduce hippocampal neurons apoptosis by increasing the $\mathrm{Bcl}-2 / \mathrm{Bax}$ ratio and inhibiting activated caspase- 3 expression (64); third, TEAS regulates serum neuron-specific enolase and $S 100-\beta$ protein $(S 100-\beta)$ levels $(28,36)$, or reduces the oxidative stress reaction (31); fourth, TEAS enhances the effect of postoperative analgesia and reduces the dosage of analgesics (65).

A recent expert consensus concluded that prevention was the best treatment for postoperative cognitive impairment and proposed some practical recommendations, such as pain control, cognitive screening, and minimizing psychoactive treatments $(12,66,67)$. However, the recommended brain assessments and simple preventive measures were not routinely implemented and need further work to identify potential strategies with high therapy compliance (68). As a new type of acupuncture therapy, TEAS has attracted the attention of many researchers because of its multiple advantages, such as unified parameters, simple operation, and good patient compliance. The results of our review provided low to moderate certainty of evidence that TEAS was beneficial for the prevention of POCD in patients undergoing anesthesia and surgery, and it significantly reduced the incidence of PONV. We also observed the benefits of TEAS in improving neurological function scores, although the evidence level was downgraded to low due to statistical heterogeneity. Low to moderate certainties of evidence suggested that TEAS could enhance postoperative analgesia and shorten hospital stay. In general, these findings may provide new strategies for the management of perioperative brain health guidelines in the future.

\section{LIMITATIONS}

This review has several limitations. First, the long-term followup results are unknown because the observation time of the included studies was generally within 7 days after surgery. Second, all 29 included studies were conducted in China and most had small sample sizes $(\leq 100)$, which may have affected the reliability and extrapolation of the overall results. Due to the small sample size of the included studies, the estimated results were likely overestimated. Consequently, we performed a subgroup analysis to explore the impact of small sample sizes by grouping their sample size into quarters. The forest plot showed that the overall pooled results were not overestimated $(p>0.05)$ (Supplementary Figure 16). Generally speaking, the meta-analysis included no trials with large sample sizes; thus, the detection strength was also limited. Third, some of the studies had high risk and low quality, which downregulated the evidence strength of the research results. Therefore, future trials with higher-quality and larger sample sizes are needed to make more firm conclusions.

\section{CONCLUSION}

Our review systematically investigated and quantified the preventive effects of TEAS on POCD. Overall, our findings suggested that the application of TEAS in the perioperative period was associated with improved cognitive function scores and reduced POCD rates in the early postoperative period. Meanwhile, limited evidence suggested that TEAS could enhance the postoperative analgesic effect within $24 \mathrm{~h}$ after surgery, decrease PONV incidence, and reduce the length of hospital stay. Large RCTs are needed to determine the preventive effects of TEAS for POCD before recommending its routine use in surgical practice. 


\section{DATA AVAILABILITY STATEMENT}

The original contributions presented in the study are included in the article/Supplementary Material, further inquiries can be directed to the corresponding author/s.

\section{AUTHOR CONTRIBUTIONS}

GH conceived the original study design. TZ and LO developed the search strategy and searched the literature database. JL and YS screened the eligible studies and extracted the data. JL and TZ evaluated the risk of bias. $\mathrm{LO}$ and ZC performed data analysis and evaluated the certainty of evidence. TZ drafted the first manuscript and GH revised the manuscript. All authors read and approved the submitted version.

\section{REFERENCES}

1. O’ Brien H, Mohan H, Hare CO, Reynolds JV, Kenny RA. Mind over matter? The hidden epidemic of cognitive dysfunction in the older surgical patient. Ann Surg. (2017) 265:677-91. doi: 10.1097/SLA.0000000000001900

2. Weiser TG, Haynes AB, Molina G, Lipsitz SR, Esquivel MM, UribeLeitz T, et al. Estimate of the global volume of surgery in 2012: an assessment supporting improved health outcomes. Lancet. (2015) 385 (Suppl 2):S11. doi: 10.1016/S0140-6736(15)60806-6

3. Alam A, Hana Z, Jin Z, Suen KC, Ma D. Surgery, neuroinflammation and cognitive impairment. Ebiomedicine. (2018) 37:54756. doi: 10.1016/j.ebiom.2018.10.021

4. Moller JT, Cluitmans P, Rasmussen LS, Houx P, Rasmussen H, Canet J, et al. Long-term postoperative cognitive dysfunction in the elderly ISPOCD1 study. Lancet. (1998) 351:857-61. doi: 10.1016/S0140-6736(97)07382-0

5. Daiello LA, Racine AM, Yun GR, Marcantonio ER, Xie Z, Kunze LJ, et al. Postoperative delirium and postoperative cognitive dysfunction: overlap and divergence. Anesthesiology. (2019) 131:477-91. doi: 10.1097/ALN.0000000000002729

6. Steinmetz J, Christensen KB, Lund T, Lohse N, Rasmussen LS. Long-term consequences of postoperative cognitive dysfunction. Anesthesiology. (2009) 110:548-55. doi: 10.1097/ALN.0b013e318195b569

7. Monk TG, Weldon BC, Garvan CW, Dede DE, van der Aa MT, Heilman $\mathrm{KM}$, et al. Predictors of cognitive dysfunction after major noncardiac surgery. Anesthesiology. (2008) 108:18-30. doi: 10.1097/01.anes.0000296071.19434.1e

8. Needham MJ, Webb CE, Bryden DC. Postoperative cognitive dysfunction and dementia: What we need to know and do. Br J Anaesth. (2017) 119:i11525. doi: 10.1093/bja/aex354

9. Silbert B, Evered L, Scott DA, McMahon S, Choong P, Ames D, et al. Preexisting cognitive impairment is associated with postoperative cognitive dysfunction after hip joint replacement surgery. Anesthesiology. (2015) 122:1224-34. doi: 10.1097/ALN.0000000000000671

10. Eckenhoff RG, Maze M, Xie Z, Culley DJ, Goodlin SJ, Zuo Z, et al. Perioperative neurocognitive disorder: State of the preclinical science. Anesthesiology. (2020) 132:55-68. doi: 10.1097/ALN.0000000000002956

11. Laalou FZ, Jochum D, Pain L. Postoperative cognitive dysfunction (POCD): strategy of prevention, assessment and management. Ann Fr Anesth Reanim. (2011) 30:e49-53. doi: 10.1016/j.annfar.2011.08.009

12. Peden CJ, Miller TR, Deiner SG, Eckenhoff RG, Fleisher LA. Improving perioperative brain health: An expert consensus review of key actions for the perioperative care team. Br J Anaesth. (2021) 126:423-32. doi: 10.1016/j.bja.2020.10.037

13. Wang H, Xie Y, Zhang Q, Xu N, Zhong H, Dong H, et al. Transcutaneous electric acupoint stimulation reduces intra-operative remifentanil consumption and alleviates postoperative side-effects in patients undergoing sinusotomy: a prospective, randomized, placebo-controlled trial. Br J Anaesth. (2014) 112:1075-82. doi: 10.1093/bja/aeu001

\section{FUNDING}

This study was supported by the National Natural Science Foundation of China (no. 81573941); Hunan Natural Science Foundation general project (no. 2020JJ4476); the Hunan University of Chinese Medicine, domestic first-class construction discipline, Open Fund Project of First-class Discipline of Hunan University of Chinese Medicine (no. 2021ZYX40); Hu Guoheng Famous Doctor Inheritance Studio.

\section{SUPPLEMENTARY MATERIAL}

The Supplementary Material for this article can be found online at: https://www.frontiersin.org/articles/10.3389/fmed. 2021.756366/full\#supplementary-material

14. Arnberger M, Stadelmann K, Alischer P, Ponert R, Melber A, Greif R Monitoring of neuromuscular blockade at the P6 acupuncture point reduces the incidence of postoperative nausea and vomiting. Anesthesiology. (2007) 107:903-8. doi: 10.1097/01.anes.0000290617.98058.d9

15. Xu M, Zhou SJ, Jiang CC, Wu Y, Shi WL, Gu HH, et al. The effects of P6 electrical acustimulation on postoperative nausea and vomiting in patients after infratentorial craniotomy. J Neurosurg Anesthesiol. (2012) 24:3126. doi: 10.1097/ANA.0b013e31825eb5ef

16. Asmussen S, Maybauer DM, Chen JD, Fraser JF, Toon MH, Przkora R, et al. Effects of acupuncture in anesthesia for craniotomy: a meta-analysis. J Neurosurg Anesthesiol. (2017) 29:219-27. doi: 10.1097/ANA.0000000000000290

17. Song B, Chang Y, Li Y, Zhu J. Effects of transcutaneous electrical acupoint stimulation on the postoperative sleep quality and pain of patients after VideoAssisted thoracoscopic surgery: a prospective, randomized controlled trial. Nat Sci Sleep. (2020) 12:809-19. doi: 10.2147/NSS.S270739

18. Page MJ, McKenzie JE, Bossuyt PM, Boutron I, Hoffmann TC, Mulrow CD, et al. The PRISMA 2020 statement: an updated guideline for reporting systematic reviews. BMJ. (2021) 372:n71. doi: 10.1136/bmj.n71

19. Caparrotta TM, Dear JW, Colhoun HM, Webb DJ. Pharmacoepidemiology: using randomised control trials and observational studies in clinical decisionmaking. Br J Clin Pharmacol. (2019) 85:1907-24. doi: 10.1111/bcp.14024

20. Freemantle N, Marston L, Walters K, Wood J, Reynolds MR, Petersen I. Making inferences on treatment effects from real world data: propensity scores, confounding by indication, and other perils for the unwary in observational research. BMJ. (2013) 347:f6409. doi: 10.1136/bmj.f6409

21. Higgins JP, Altman DG, Gotzsche PC, Juni P, Moher D, Oxman AD, et al. The Cochrane Collaboration's tool for assessing risk of bias in randomised trials. BMJ. (2011) 343:d5928. doi: 10.1136/bmj.d5928

22. Guyatt GH, Oxman AD, Vist GE, Kunz R, Falck-Ytter Y, AlonsoCoello P, et al. GRADE: an emerging consensus on rating quality of evidence and strength of recommendations. BMJ. (2008) 336:9246. doi: 10.1136/bmj.39489.470347.AD

23. Dechartres A, Trinquart L, Boutron I, Ravaud P. Influence of trial sample size on treatment effect estimates: meta-epidemiological study. BMJ. (2013) 346:f2304. doi: 10.1136/bmj.f2304

24. Borenstein M, Hedges LV, Higgins JP, Rothstein HR. A basic introduction to fixed-effect and random-effects models for meta-analysis. Res Synth Methods. (2010) 1:97-111. doi: 10.1002/jrsm.12

25. Sterne JA, Sutton AJ, Ioannidis JP, Terrin N, Jones DR, Lau J, et al. Recommendations for examining and interpreting funnel plot asymmetry in meta-analyses of randomised controlled trials. BMJ. (2011) 343:d4002. doi: 10.1136/bmj.d4002

26. Shi L, Lin L. The trim-and-fill method for publication bias: practical guidelines and recommendations based on a large database of metaanalyses. Medicine. (2019) 98:e15987. doi: 10.1097/MD.00000000000 15987 
27. Ren Q. The effect of transcutaneous acupoint electrical stimulation on the cerebral injury from neurosurgery procedure (dissertation/master's thesis). Wenzhou Medical College, Wenzhou, China. (2007).

28. Zhou B, Ni Y, Qu Q, Dai W, Ye X, Xiang D, et al. Effect of controlled hypotension combined with transcutaneous electrical acupoint stimulation on early postoperative cognitive dysfunction in spinal surgery. Chin J Surg Integr Tradit West Med. (2011) 17:476-8. Available online at: https://d.wanfangdata.com.cn/periodical/ChlQZXJpb2RpY2FsQ0hJTmV3Uz IwMjExMTE2EhR6Z3p4eWpod2t6ejIwMTEwNTAwORoIdXN0ZTFjcGw\% $3 \mathrm{D}$

29. Lin S, Gao J, Yin Z, Zhou L, Chen X. Impact of the different frequencies of electroacupuncture on cognitive function in patients after abdominal operation under combined anesthesia of acupuncture and drugs. Chin Acup Moxib. (2013) 33:1109-12.

30. Wu Y, Yuan J. Effects of transcutaneous electrical acupoint stimulation combined with inhalation anesthesia on postoperative cognitive function of aged patients with laparoscopic cholecystectomy. China J Endoscopy. (2014) 20:247-51. Available online at: https://d.wanfangdata.com.cn/periodical/ChlQZXJpb2RpY2FsQ0hJTmV3Uz IwMjExMTE2Eg96Z25qenoyMDE0MDMwMDYaCDh4OWpkMXo1

31. Ni J, Jiang L, Zhou Y, Mo Y, Wu Q, Wang J. Effect of transcutaneous acupoint electrical stimulation on postoperative cognitive function in elderly patients undergoing laparoscopic resection of rectal cancer. Chin Gen Prac. (2015) 18:1390-5. Available online at: https://d.wanfangdata.com.cn/periodical/ChlQZXJpb2RpY2FsQ0hJTmV3Uz IwMjExMTE2Eg96Z3FreXgyMDE1MTIwMTEaCGhuNG9jMnR1

32. Yin Z, Meng Z, Lin S, Gao J, Chen X. Impacts of electrical acupoint stimulation on postoperative cognitive dysfunction and inflammation cytokines in elderly patients. Chin J Acupunct Moxib. (2015) 4:159-63.

33. Yang Z, Zhang Y, Tong B, Zheng R, Guo Y. Effect of TEAS combined with general anesthesia on cognitive function of patients undergoing gynaecological laparoscopy. J Shanxi Coll Tradit Chin Med. (2015) 38:38-42. Available online at: https://d.wanfangdata.com.cn/periodical/ChlQZXJpb2RpY2FsQ0hJTmV3Uz IwMjExMTE2EhFzeHp5eHl4YjIwMTUwNTAxNRoIYzI3dDFubHE\%3D

34. Wang D, Peng C, Ma T, Hu J. Effect of transcutaneous acupoint electrical stimulation on postoperative cognitive function in elderly patients with artificial femoral head replacement. Chin Arch Tradit Chin Med. (2016) 34:431-3. Available online at: https://d.wanfangdata.com.cn/periodical/ChlQZXJpb2RpY2FsQ0hJTmV3Uz IwMjExMTE2Eg56eXl4azIwMTYwMjA1MhoIbWNydjQ2ZGg\%3D

35. Zhu J, Teng X, Yang Y, Wan Y, Li Y. Effects of transcutaneous acupoint electrical stimulation and dexmedetomidine on postoperative cognitive dysfunction in patients underwent laparoscopic surgery. J China Med Univ. (2016) 45:345-8. Available online at: https://d.wanfangdata.com.cn/periodical/ChlQZXJpb2RpY2FsQ0hJTmV3Uz IwMjExMTE2EhF6Z3lrZHh4YjIwMTYwNDAxNBoIams5YnBjYXg\%3D

36. Wei $\mathrm{W}$, Teng $\mathrm{X}$, Yang $\mathrm{Y}$, Huang $\mathrm{X}$, Zhu J. Effect of transcutaneous electrical stimulation at acupoints on postoperative cognitive function of gynecological laparoscopy patients. Chin Arch Trad Chin Med. (2016) 34:1186-9. Available online at: https://d.wanfangdata.com.cn/periodical/ChlQZXJpb2RpY2 FsQ0hJTmV3UzIwMjExMTE2Eg56eXl4azIwMTYwNTA0NxoIZnFlOHhpZX $\mathrm{Q} \% 3 \mathrm{D}$

37. Li X, Wei W, Teng X, Yang Y, Huang X, Huang J. Effect of transcutaneous electrical acupoint stimulation on postoperative cognitive function in patients undergoing radical thoracoscopic lung cancer operation. Chin Arch Tradit Chin Med. (2016) 34:1752-5. Available online at: https://d.wanfangdata.com.cn/periodical/ChlQ ZXJpb2RpY2FsQ0hJTmV3UzIwMjExMTE2Eg56eXl4azIwMTYwNzA2MxoI OW1qY2tncXI\%3D

38. Wang D, Ma T, Zhou C, Zhu H. Clinical observation of transcutaneous acupoint electrical stimulation in the treatment of cognitive dysfunction in elderly patients after abdominal surgery under general anesthesia. Zhejiang J Integr Tradit Chin West Med. (2016) 26:732-5. Available online at: https://d.wanfangdata.com.cn/periodical/ChlQZ XJpb2RpY2FsQ0hJTmV3UzIwMjExMTE2EhJ6anp4eWpoenoyMDE2MDgwM TcaCDZ3ZXN5NHhu
39. Zhao L, Wang S, Wang B. Effects of transcutaneous electrical acupoint stimulation on early postoperative cognitive function in patients with transsphenoidal surgery. Chin Med Herald. (2017) 14:12-5. Available online at: https://d.wanfangdata.com.cn/periodical/ ChlQZXJpb2RpY2FsQ0hJTmV3UzIwMjExMTE2Eg95eWN5engyMDE3MD EwMDQaCGd3NHJxeHZu

40. Wei W, Teng $\mathrm{X}$, Yang $\mathrm{Y}, \mathrm{Li}$ X, Zhu J. Effect of different frequency transcutaneous electrical acupoint stimulation treated on the postoperative cognitive function of patients undergoing gynecological laparoscopy. Prog Mod Biomed. (2017) 17:155-8. Available online at: https://d.wanfangdata.com.cn/periodical/ChlQZX Jpb2RpY2FsQ0hJTmV3UzIwMjExMTE2Eg1zd2N4MjAxNzAxMDQwGgh5N jdvcmd0OQ\%3D\%3D

41. Tan X. Effect of TEAS on cognitive function and serum S100 beta protein in senile patients after cholecystectomy. J Clin Acupunct Moxib. (2017) 33:9-12. Available online at: https://d.wanfangdata.com.cn/periodical/ChlQZXJpb2RpY2FsQ0hJTmV3Uz IwMjExMTE2Eg96amxjenoyMDE3MDcwMDMaCHFhOTd0OG84

42. Tang Y, Qing M. Effect of transcutaneous acupoint electrical stimulation on postoperative cognitive function in elderly patients with colorectal cancer. Mod Med Heal. (2017) 33:3623-5. Available online at: https://d.wanfangdata.com.cn/periodical/ChlQZX Jpb2RpY2FsQ0hJTmV3UzIwMjExMTE2Eg94ZHl5d3MyMDE3MjMwMzIaC DZvNG84cnN1

43. Fan X, Zhang F, Huang L, Xiao H, Jiang K, Hao L. Effects of TEAS combined with general anesthesia versus epidural block combined with general anesthesia on recovery of patients undergoing laparoscopic radical resection of colorectal cancer. Chin J Anesthesiol. (2018) 38:1053-7. Available online at: https://d.wanfangdata.com.cn/periodical/ ChlQZXJpb2RpY2FsQ0hJTmV3UzIwMjExMTE2EhJ6aG16eHp6OTgyMDE4 MDkwMDgaCDF4eHZhOWYy

44. Mi Z, Gao J, Chen X, Ge Y, Lu K. Effects of transcutaneous electrical acupoint stimulation on quality of recovery during early period after laparoscopic cholecystectomy. Chin Acup Moxib. (2018) 38:256-60. doi: $10.13703 /$ j.0255-2930.2018.03.007

45. Sun K, Liang L, Hu X, Wang G. Effects of early transcutaneous acupoint electrical stimulation on postoperative cognition of elderly patients with hip fractures. Chin J Med Phys. (2018) 35:10759. Available online at: https://d.wanfangdata.com.cn/periodical/ ChlQZXJpb2RpY2FsQ0hJTmV3UzIwMjExMTE2EhJ6Z314d2x4enoyMDE4M DkwMTUaCG9ka2MzbWVk

46. Mao H, Li S, Li H, Chen Y. Effect of transcutaneous acupoint electrical stimulation on early recovery of patients with breast cancer after operation. Jinagsu Med J. (2019) 45:98-9. Available online at: https://d.wanfangdata.com.cn/periodical/ChlQ ZXJpb2RpY2FsQ0hJTmV3UzIwMjExMTE2Eg1qc315MjAxOTAxMDMyGgg 2NzFseWJxYw\%3D\%3D

47. Li S, Yang J. Influence of percutaneous acupoints electrical stimulation on postoperative cognitive function and immune function in patients after laparoscopic myomectomy. Chin J Pract Med. (2019) 46:61-4. doi: 10.3760/CMA.J.ISSN.1674-4756.2019.03.018

48. Wu X, Chen $\mathrm{W}$. Effects of transcutaneous acupoint electrical stimulation assisted with general anesthesia on postoperative immune function and cognitive function in elderly patients undergoing cardiac surgery. Chin Med Herald. (2019) 16:145-57. Available online at: https://d.wanfangdata.com.cn/periodical/ChlQZX Jpb2RpY2FsQ0hJTmV3UzIwMjExMTE2Eg95eWN5engyMDE5MDkwMzka CGs4d2RscW5z

49. Duan C, Cui Z, Zhang X, Wen R, Li X. Effect of transcutaneous electrical acupoint stimulation on postoperative cognitive dysfunction in elderly patients. Hainan Med J. (2019) 30:20714. Available online at: https://d.wanfangdata.com.cn/periodical/ ChlQZXJpb2RpY2FsQ0hJTmV3UzIwMjExMTE2EhFoYWluYW55eDIwMT kxNjAxMBoIdHk1NGdlbXk\%3D

50. Lu B, Ding L, Wang Q, Ke H, Yu B. Effect of transcutaneous electrical acupoint stimulation on cerebral oxygen metabolism, postoperative cognitive function and analgesic effect in elderly patients undergoing hip replacement. Prog Mod Biomed. (2019) 19:3094-114. 
51. Yu X, Zhang F, Chen B. The effect of TEAS on the quality of early recovery in patients undergoing gynecological laparoscopic surgery: a prospective, randomized, placebo-controlled trial. Trials. (2020) 21:43. doi: 10.1186/s13063-019-3892-4

52. Yang T. The effect of transcutaneous acupoint electrical stimulation combined with dexmedetomidine on the cognitive function of elderly patients after hip surgery (dissertation/master's thesis). Xinjiang Medical University, Wulumuqi, China. (2020).

53. Huang $\mathrm{X}$, Zhang J, Luo $\mathrm{T}$, Wei C, Wu A. Effect of transcutaneous electrical acupoint stimulationon on postoperative neurocognitive disorders in patients undergoing off-pump coronary artery bypass grafting. J Clin Anesthesiol. (2020) 36:861-5. Available online at: https://d.wanfangdata.com.cn/periodical/ChlQZXJpb2RpY2FsQ0hJTmV3U zIwMjExMTE2EhBsY216eHp6 MjAyMDA5MDA2GghzOGN4cGw2Zw\%3D $\% 3 \mathrm{D}$

54. Wu Y, Luo $H$. Effect of electrical acupoint stimulation on postoperative cognitive dysfunction after total intravenous anesthesia. Shanghai J Acupunct Moxib. (2020) 39:1161-5. Available online at: https://d.wanfangdata.com.cn/periodical/ChlQZ XJpb2RpY2FsQ0hJTmV3UzIwMjExMTE2Eg9zaHpqenoyMDIwMDkwMTY aCG12Nnhib2Uy

55. Duan C, Xun S, Zhang X, Jiao M, Guo X. Effects of transcutaneous electrical acupoint stimulation pretreatment on postoperative cognitive function and inflammatory factors in elderly patients. J Ningxia Med Univ. (2021) 43:43-7. Available online at: https://d.wanfangdata.com.cn/periodical/ /ChlQZXJpb2RpY2FsQ0hJTmV3UzIwMjExMTE2EhBueHl4eXhiMjAyMTA xMDA4GghvMzFiZm9qZw\%3D\%3D

56. Tan R, He Y, Zhang S, Pu D, Wu J. Effect of transcutaneous electrical acupoint stimulation on protecting against radiotherapy- induced ovarian damage in mice. J Ovarian Res. (2019) 12:65. doi: 10.1186/s13048-019-0541-1

57. Selwood A, Orrell M. Long term cognitive dysfunction in older people after non-cardiac surgery. BMJ. (2004) 328:120-1. doi: 10.1136/bmj.328.7432.120

58. Mason SE, Noel-Storr A, Ritchie CW. The impact of general and regional anesthesia on the incidence of post-operative cognitive dysfunction and postoperative delirium: a systematic review with meta-analysis. J Alzheimers Dis. (2010) 22 (Suppl 3):67-79. doi: 10.3233/JAD-2010-101086

59. Miller D, Lewis SR, Pritchard MW, Schofield-Robinson OJ, Shelton $\mathrm{CL}$, Alderson $\mathrm{P}$, et al. Intravenous versus inhalational maintenance of anaesthesia for postoperative cognitive outcomes in elderly people undergoing non-cardiac surgery. Cochrane Database Syst Rev. (2018) 8:D12317. doi: 10.1002/14651858.CD012317.pub2

60. Hughes CG, Patel MB, Jackson JC, Girard TD, Geevarghese SK, Norman $\mathrm{BC}$, et al. Surgery and anesthesia exposure is not a risk factor for cognitive impairment after major noncardiac surgery and critical illness. Ann Surg. (2017) 265:1126-33. doi: 10.1097/SLA.0000000000001885

61. Chen J, Zhang Y, Li X, Wan Y, Ji X, Wang W, et al. Efficacy of transcutaneous electrical acupoint stimulation combined with general anesthesia for sedation and postoperative analgesia in minimally invasive lung cancer surgery: a randomized, double-blind, placebo-controlled trial. Thorac Cancer. (2020) 11:928-34. doi: 10.1111/1759-7714.13343

62. Mu L, Gao H, Zhao ML, Ren HF, Ma HS. Effect of transcutaneous electrical acupoint stimulation on recovery of gastrointestinal function after cesarean section. Chin Acup Moxib. (2019) 39:259-62. doi: 10.13703/j.0255-2930.2019.03.010

63. Chen J, Tu Q, Miao S, Zhou Z, Hu S. Transcutaneous electrical acupoint stimulation for preventing postoperative nausea and vomiting after general anesthesia: a meta-analysis of randomized controlled trials. Int J Surg. (2020) 73:57-64. doi: 10.1016/j.ijsu.2019.10.036

64. Liu Z, Shen Y, Lian L, Shao X, Fang J. Inflammatory-modulating mechanism of transcutaneous electrical acupoint stimulation combined with general anesthesia or controlled hypotension on postoperative hippocampal neuroprotection. Chin Acup Moxib. (2013) 33:149-55.

65. Liang J, Zheng L, Wang G, Wu H, Wang L. Effects of transcutaneous electric stimulation of acupuncture points on perioperative T-lymphocyte immune function and postoperative analgesia in patients undergoing radical rectal cancer operation. Chin J Anesthesiol. (2008) 28:20810. Available online at: https://d.wanfangdata.com.cn/periodical/ ChlQZXJpb2RpY2FsQ0hJTmV3UzIwMjExMTE2EhJ6aG16eHp6OTgyMDA 4MDMwMDQaCGVsYTh3ZHox

66. Mohanty S, Rosenthal RA, Russell MM, Neuman MD, Ko CY, Esnaola NF. Optimal perioperative management of the geriatric patient: a best practices guideline from the American college of surgeons NSQIP and the American geriatrics society. J Am Coll Surg. (2016) 222:93047. doi: 10.1016/j.jamcollsurg.2015.12.026

67. Berger M, Schenning KJ, Brown CT, Deiner SG, Whittington RA, Eckenhoff $\mathrm{RG}$, et al. Best practices for postoperative brain health: recommendations from the fifth international perioperative neurotoxicity working group. Anesth Analg. (2018) 127:1406-13. doi: 10.1213/ANE.00000000000 03841

68. Deiner S, Fleisher LA, Leung JM, Peden C, Miller T, Neuman MD. Adherence to recommended practices for perioperative anesthesia care for older adults among US anesthesiologists: results from the ASA Committee on Geriatric Anesthesia-Perioperative Brain Health Initiative ASA member survey. Perioper Med (Lond). (2020) 9:6. doi: 10.1186/s13741-0200136-9

Conflict of Interest: The authors declare that the research was conducted in the absence of any commercial or financial relationships that could be construed as a potential conflict of interest.

Publisher's Note: All claims expressed in this article are solely those of the authors and do not necessarily represent those of their affiliated organizations, or those of the publisher, the editors and the reviewers. Any product that may be evaluated in this article, or claim that may be made by its manufacturer, is not guaranteed or endorsed by the publisher.

Copyright (C) 2021 Zhang, Ou, Chen, Li, Shang and Hu. This is an open-access article distributed under the terms of the Creative Commons Attribution License (CC $B Y)$. The use, distribution or reproduction in other forums is permitted, provided the original author(s) and the copyright owner(s) are credited and that the original publication in this journal is cited, in accordance with accepted academic practice. No use, distribution or reproduction is permitted which does not comply with these terms. 